\title{
Construção de um banco de termos da linguagem especial de enfermagem ${ }^{1}$
}

\author{
Terminology bank of nursing language
}

\section{Construccion del banco de terminos del lenguaje especial de la enfermería}

\section{Meire Chucre Tannure ${ }^{\mathrm{I}}$, Tânia Couto Machado Chianca ${ }^{\mathrm{II}}$, Telma Ribeiro GarciaIII}

\begin{abstract}
1 Trabalho vinculado ao Projeto de Pesquisa "Sistematização da Assistência de Enfermagem em unidades de terapia intensiva com a utilização de um software", financiado pelo CNPq. Aprovado pelo Comitê de Ética e Pesquisa da UFMG - Parecer n 565/06.

${ }^{I}$ Enfermeira Intensivista. Mestre em Enfermagem. Doutoranda do Programa de Pós-Graduação em Enfermagem Escola de Enfermagem da Universidade Federal de Minas Gerais (EE/UFMG). Docente da PUC Minas e da Pós-Graduação da Faculdade Pitágoras e Instituto de Educação Continuada da PUC Minas. Belo Horizonte, MG. E-mail: meirechucre@yahoo.com.br.

II Enfermeira. Doutora em Enfermagem. Professor Associado da EE/UFMG. Professora do Programa de Pós-Graduação em Enfermagem da EE/UFMG. Belo Horizonte, MG. E-mail: tchianca@enf.ufmg.br.

III Enfermeira. Doutora em Enfermagem. Professora Adjunto IV do Departamento de Enfermageme Saúde Pública e Psiquiatria do Centro de Ciências da Saúde da Universidade Federal da Paraíba (CCS/UFPB). Professora do Programa de Pós-Graduação em Enfermagem da CCS-UFPB. Pesquisadora CNPq. João Pessoa, PB. E-mail: tegarcia@ccs.ufpb.br.
\end{abstract}

\section{RESUMO}

Este artigo apresenta a metodologia empregada na construção de um banco de termos da linguagem especial de Enfermagem descrevendo o processo de extração e padronização de termos identificados em prontuários de pacientes internados em uma unidade de tratamento intensivo de adultos, mapeando os termos extraídos com os termos existentes na CIPE $®$ - versão 1.0 e classificando os termos não constantes nos sete eixos dessa classificação. É possível identificar termos que são utilizados na prática por enfermeiros e construir bancos de termos que possam alimentar sistemas de informação que auxiliem na operacionalização das etapas do processo de Enfermagem e na construção de catálogos CIPE $®$.

Descritores: Classificação; Terminologia; Enfermagem.

\section{ABSTRACT}

This article presents the methodology which was used in the building of a terminology bank of specific nursing language. The process of extraction was described as well as the standardization of terms identified in ICUadult patients' clinical records, matching the extracted terms with the ones included in ICNP ${ }^{\circledR}$-Version 1.0 and, classifying those not included in the 7 axes of classification. It is possible to identify terms that are used in nursing clinical practice and to build a terminology bank that can feed information systems which may aid nursing process stages implementation and CIPE $®$ catalogues building.

Descriptors: Classification; Terminology; Nursing.

\section{RESUMEN}

En este artículo presentamos la metodología empleada para la construcción de un banco de términos del lenguaje especial de la enfermería describiendo el proceso de extracción y uniformización de términos identificados en la historia clínica de los pacientes internados en una unidad de tratamiento intensivo de adultos, mapeando los términos extraídos con los términos existentes en la CIPE ${ }^{\circ}$-versión 1.0, clasificando los términos que no constan en los siete ejes de esa clasificación. Es posible identificar los términos que son utilizados en la práctica por los enfermeros y construir bancos de términos que puedan alimentar sistemas de información que ayuden en la funcionalidad de las etapas del proceso de enfermería y en la construcción de catálogos CIPE®.

Descriptores: Clasificación; Terminología; Enfermería. 


\section{INTRODUÇÃO}

A prática de Enfermagem vem demonstrando, ao longo dos anos, a necessidade de construção de um vocabulário próprio, preciso e consensual, que não só permita a sua definição como ciência, mas que proporcione uma aplicação mais eficaz de seus princípios, métodos e técnicas, o que viria a enriquecer sobremaneira essa área do conhecimento humano(1).

A falta de uma linguagem universal, que estabeleça a definição e descrição da prática profissional, tem levado enfermeiros a se inquietarem e a demonstrarem seu compromisso com o desenvolvimento da Enfermagem como ciência(2-3).

Uma ciência é caracterizada pela universalização de sua linguagem. Mas, para que isso ocorra, tornase necessário o desenvolvimento de uma terminologia própria. Uma ciência começa a existir quando seus conceitos são aceitos e a denominação dos seus fenômenos específicos começa a ser realizada(3).

A utilização de sistemas de classificação permite que sejam capturados dados e informações relevantes para identificar os resultados obtidos com as intervenções de Enfermagem e permite, também, que haja a comparação entre as práticas realizadas em nível nacional e internacional(4). As várias tentativas de identificar e denominar os elementos que descrevem a prática da Enfermagem está registrado na literatura especializada, podendo ser citada, como grande contribuição para isso, o fato de, no início da década de 1950, enfermeiras começarem a identificar os conceitos específicos da profissão(1).

Temos, hoje, à nossa disposição, vários sistemas de classificação dos elementos da prática de Enfermagem - de diagnósticos, resultados e intervenções - que podem ser utilizadas durante a implementação das etapas do processo de Enfermagem $(\mathrm{PE})^{(5)}$.

Estes sistemas estão em constante evolução e, para que esse aprimoramento ocorra, existe a necessidade de formulação de conceitos e avaliação constante dos mesmos, uma vez que eles compõem essas terminologias e representam o ponto de partida para o desenvolvimento do trabalho terminológico. Esse processo é considerado teórico e tem, como maior resultado, a construção do conhecimento, essencial para o desenvolvimento da Enfermagem como profissão e ciência(6-8).

A elaboração de sistemas de classificação da linguagem da Enfermagem pressupõe, de modo implícito ou explícito, que os profissionais da área constituem um grupo sócio profissional, que, em seu ambiente de trabalho, utiliza um vocabulário técnico particular a que se pode denominar linguagem especial da Enfermagem ${ }^{(1)}$.

Essa linguagem é composta por uma terminologia, definida como um conjunto de palavras técnicas pertencentes a uma ciência e que, em um sentido mais restrito e especializado, pode designar o estudo científico dos conceitos e termos utilizados nas especialidades ${ }^{(9)}$.

Os conceitos dos termos usados nesta linguagem especializada são considerados unidades básicas do pensamento teórico, capazes de representar a realidade e facilitar a comunicação(10).

A Enfermagem vem utilizando uma linguagem representada por termos clínicos que integram o contexto teórico e prático da profissão, e o estudo dos conceitos destes termos pode possibilitar a melhora da comunicação profissional( ${ }^{(3,8)}$. Sendo assim, qualquer que seja a forma como são expressos esses elementos da prática, eles são considerados relevantes e podem ser denominados componentes primários de uma classificação(11).

Houve, nas últimas décadas, um aumento na produção de sistemas de classificação na Enfermagem, a maioria deles desenvolvidos nos Estados Unidos da América (EUA), com um crescente interesse de seus autores na tradução e aplicação dos mesmos em outras culturas(11). Dentre estes sistemas, alguns já são conhecidos e utilizados por enfermeiros brasileiros, como o da Associação NorteAmericana de Diagnósticos de Enfermagem (North American Nursing Diagnosis Association - NANDA); Classificação de Intervenções de Enfermagem (Nursing Interventions Classification - NIC); Classificação de Resultados de Enfermagem (Nursing Outcomes Classification - NOC); Classificação de Cuidados Domiciliares de Saúde (Home Health Care Classification - HHCC); Sistema de Cuidados Comunitários de Omaha (The Omaha System); Grupo de Dados de Enfermagem Perioperatória (Perioperative Nursing Data Set - PNDS) e a Classificação Internacional para a Prática de Enfermagem $\left(\mathrm{CIPE}^{\circledR}\right)^{(12-13)}$.

A utilização dessas classificações favorece a comparação do conhecimento globalmente. Se o vocabulário utilizado na prática se fizer de forma padronizada poderá haver uma melhor compreensão por parte dos profissionais quanto ao monitoramento da qualidade do cuidado de Enfermagem ${ }^{(8)}$ podendo, desse modo, favorecer a obtenção de informações valiosas sobre a contribuição da Enfermagem para os pacientes $^{(14)}$.

Cabe, no entanto ressaltar que não existem classificações específicas para todas as áreas de atuação do profissional do enfermeiro. Porém, sabese que 0 Conselho Internacional de Enfermeiros (CIE), responsável pela construção da CIPE $^{\circledR}$, tem o interesse de recolher e codificar termos utilizados pela enfermagem em áreas específicas, organizandoos e criando catálogos $\operatorname{CIPE}^{\circledR(15)}$.

Deste modo, a identificação da linguagem utilizada por enfermeiros em áreas específicas e o mapeamento dos termos identificados com os termos 
da CIPE $^{\circledR}$ - Versão 1.0 se faz necessária. Para tanto, torna-se relevante compartilhar estratégias metodológicas que vêm sendo realizadas em pesquisas com essa finalidade. Assim, tendo por base atender essa demanda, o objetivo desse trabalho é descrever a metodologia empregada para construir um banco de termos da linguagem especial de enfermagem. Neste sentido, optou-se pela apresentação de como está composta a $\operatorname{CIPE}^{\circledR}$-Versão 1.0, processo de extração de termos de prontuários, eliminação de repetições, de normalização de termos, mapeamento cruzado entre os termos extraídos dos prontuários e os termos constantes na classificação e o procedimento de refinamento de termos.

\section{Composição da CIPE $^{\circledR}$ - Versão 1.0.}

A CIPE ${ }^{\circledR}$-Versão 1.0 contém um simplificado modelo único de sete eixos, e pode ser considerado um recurso capaz de acomodar vocabulários atuais e novos termos, utilizando-se de uma terminologia composicional(15).

Assim, a partir do modelo de sete eixos, os enfermeiros podem construir os enunciados dos diagnósticos, intervenções e resultados de enfermagem de acordo com as demandas detectadas nos pacientes sob seus cuidados. Os sete eixos da CIPE $^{\circledR}$ - Versão 1.0 e suas definições ${ }^{(15)}$ são:

- Foco: área de atenção relevante para a enfermagem.

- Julgamento: opinião clínica, determinação relacionada ao foco da prática profissional de enfermagem.

- Meios: forma ou método de concretizar uma intervenção.

- Ação: processo intencional aplicado a, ou desempenhado por um cliente.

- Tempo: o ponto, período, instante, intervalo ou duração de uma ocorrência.

- Localização: orientação anatômica ou espacial de um diagnóstico ou intervenção.

- Cliente: sujeito a quem o diagnóstico se refere e que é o beneficiário da intervenção.

Por ser uma classificação multiaxial, constituída por sete eixos, há a possibilidade de haver uma combinação dos conceitos dos distintos eixos, o que pode proporcionar uma maior solidez à classificação e diversificar a expressão de seus conceitos(16). Além disso, há a possibilidade da classificação ser utilizada na implementação de várias teorias de enfermagem ${ }^{(17)}$.

O CIE complementa que como essa versão da classificação foi desenvolvida como um sistema unificado da linguagem de enfermagem e uma terminologia composicional, os enfermeiros podem cruzá-la com sistemas de classificação locais, regionais ou nacionais e, assim, fazer um mapeamento da língua própria da especialidade daquela localidade com os da Classificação(15).

\section{Processo de extração de termos de prontuários e eliminação das repetições}

O ponto de partida para a realização do processo de extração de termos da linguagem especial da Enfermagem de prontuários deve ser selecionar uma instituição na qual os enfermeiros realizem o registro diário das demandas apresentadas pelos pacientes e as ações realizadas para minimizá-las. Desse modo, fazer a opção por instituições nas quais os enfermeiros realizem as etapas do PE é uma estratégia que auxilia na obtenção de termos de áreas de especialidade de Enfermagem.

Além disso, deve-se obter a autorização da diretoria da instituição de saúde, da gerencia de enfermagem e a aprovação de um Comitê de Ética em Pesquisa para que dados de prontuários de pacientes sejam manipulados, atendendo desse modo a normatização prevista na Resolução 196/96 do Conselho Nacional de Saúde, que regulamenta a pesquisa com seres vivos.

Esse processo de extração de termos é um procedimento árduo que requer atenção e cuidado por parte do profissional que o executa. Deve-se, em todas as etapas do processo, adotar uma diretriz que possa ser utilizada por todos os profissionais que forem realizar a extração a fim de que haja uniformização das estratégias utilizadas na extração de termos e para que não se perca dados relevantes.

Os termos extraídos dos prontuários devem ser transcritos em uma ficha terminológica para a construção do corpus de análise ${ }^{(9)}$ que deverá ser arquivado para posterior avaliação quanto ao contexto no qual o termo foi extraído e, assim, auxiliar na categorização do mesmo segundo os sete eixos da CIPE $^{\circledR}$ - Versão 1.0. Os dados da ficha terminológica também devem ser utilizados para alimentar uma planilha do Excel for Windows a fim de serem excluídas as repetições ${ }^{18}$, o que é realizado com o auxílio do programa.

\section{Processo de normalização de termos}

Após a eliminação das repetições, o próximo passo é submeter os termos a correções de ortografia, análise de sinonímia, adequação de tempos verbais, uniformização de gênero (feminino, masculino) e número (singular, plural) e exclusão de expressões pseudoterminológicas ${ }^{(1,8)}$. Na análise dos termos obtidos é comum identificar-se erros de ortografia que precisam ser corrigidos. Um termo digitado erroneamente não é considerado pelo sistema como um termo idêntico ao termo redigido corretamente e, deste modo, o programa não o exclui. Sendo assim, durante o trabalho terminológico deve-se ficar atento à digitação dos termos incluindo até mesmo a padronização do tamanho de fontes e 
espaçamento entre termos compostos a fim de que sejam digitados mantendo um padrão comum e uma ortografia correta visando sua exclusão durante a utilização da ferramenta de exclusão de termos repetidos do Excel for Windows ${ }^{(18)}$.

Além da correção ortográfica deve-se também eliminar as sinonímias. Durante a realização de uma pesquisa terminológica, os termos identificados devem ser atualizados, a fim de se distinguirem os sinônimos que designam o uso real de um conceito e serem excluídas as sinonímias ${ }^{(9)}$. O sistema não exclui sinônimos. Isso cabe ao terminólogo durante o trabalho de normalização dos termos. Outra ação necessária durante esse processo é a adequação dos tempos verbais, gênero e número para se evitar que termos semelhantes sejam mantidos na planilha por estarem redigidos em um tempo verbal, número ou gênero diferente.

Cabe ainda ressaltar que a extração de termos gera uma grande quantidade de expressões pseudoterminológicas definidas como elementos que ocorrem de forma casual no discurso, mas que não designam conceitos particulares, sendo considerados "lixo terminológico"(9) devendo, portanto, ser eliminadas da listagem de termos.

\section{Processo de mapeamento cruzado entre termos extraídos e os termos constantes na CIPE $^{\circledR}$ Versão 1.0}

Após a normalização dos termos o próximo passo é realizar o mapeamento cruzado entre os termos extraídos dos prontuários e já submetidos ao processo de normalização e os termos constantes na CIPE ${ }^{\circledR}$-Versão $1.0^{(7)}$.

O mapeamento cruzado é um método que pode ser útil na análise de termos não incluídos em linguagens padronizadas de enfermagem, para compará-los aos constantes em classificações de enfermagem, utilizando-se de uma terminologia uniforme ${ }^{(19)}$.

Para a realização do mapeamento cruzado, deve-se utilizar duas planilhas no programa Excel for Windows, uma contendo os termos extraídos dos prontuários e normalizados e outra contendo os termos da $\mathrm{CIPE}^{\circledR}$-Versão $1.0^{(18)}$. Desse modo, anteriormente ao procedimento o pesquisador deve alimentar uma planilha do Excel for Windows com os termos existentes na CIPE ${ }^{\circledR}$-Versão $1.0^{(18)}$.

A seguir deve-se realizar o cruzamento dos dados das duas planilhas utilizando o sistema a fim de identificar os termos em comum (termos dos prontuários existentes na classificação) e os não comuns (termos dos prontuários não existentes na classificação) ${ }^{(18)}$.

Após esse processo os termos identificados nos prontuários não constantes na classificação devem ser submetidos a um processo de refinamento a fim de serem excluídos termos pertencentes a outras áreas.

\section{Procedimento de refinamento de termos}

Dentre os termos identificados como não existentes na Classificação pode-se identificar termos pertencentes a outras áreas do conhecimento como termos da área médica e que demonstram atividades executadas pelo profissional médico, logo alguns destes termos não são considerados como representativos da área de atuação da enfermagem. Durante um trabalho terminológico, deve-se ficar atento à extração de termos pertencentes a outras áreas do conhecimento. Os termos que não forem específicos da área em estudo deverão ser eliminados da listagem ${ }^{(9)}$.

Deste modo, após o mapeamento cruzado devese eliminar termos pertencentes a outras áreas que não da enfermagem. Após a exclusão de termos de outras áreas deve-se proceder à exclusão de termos encontrados nos conceitos de termos constantes na

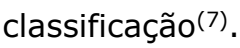

Alguns dos termos constantes nos eixos da CIPE ${ }^{\circledR}$-Versão 1.0 apresentam conceituações e, desse modo, apresentam especificações que os caracterizam. Essas características específicas dos termos devem ser incluídas em uma outra planilha Excel for Windows a fim de ser realizado um novo cruzamento, porém nesse momento utilizar-se-á a planilha de termos que representem a área da Enfermagem não constantes na $\mathrm{CIPE}^{\circledR}$-Versão 1.0 e a planilha de termos extraídos dos conceitos de termos existentes na Classificação(18).

Após esse cruzamento de dados deve-se eliminar do banco de termos aqueles constantes nos conceitos de termos da CIPE $^{\circledR}$-Versão 1.0. Finalizada essa etapa metodológica devem-se classificar os termos não constantes na $\mathrm{CIPE}{ }^{\circledR}$-Versão 1.0 segundo os sete eixos nela existentes. Para realizar essa classificação dos termos não constantes na $\mathrm{CIPE}^{\circledR}$ Versão 1.0 o pesquisador deve se direcionar pela conceituação apresentada para cada eixo pelo $\operatorname{CIE}^{(18)}$. Analisar a árvore terminológica de cada um dos sete eixos da Classificação é outra estratégia que auxilia na classificação dos termos e deve ser levada em consideração(18).

Durante este processo podem existir dúvidas e torna-se necessária a validação da alocação dos termos por outros enfermeiros com experiência e domínio acerca dos termos apresentados na $\mathrm{CIPE}^{\circledR}$ Versão 1.0 e suas definições.

\section{CONSIDERAÇÕES FINAIS}

É possível identificar termos utilizados na prática por enfermeiros e construir um banco de termos para alimentar sistemas de informação que auxiliem na operacionalização das etapas do PE. 
Este trabalho descreve uma alternativa metodológica que pode ser empregada na construção de um banco de termos da linguagem especial da Enfermagem, ou seja, termos utilizados em áreas específicas do atendimento de Enfermagem e que poderá contribuir para a determinação do que a Enfermagem identifica, faz e avalia em seus clientes.

Pesquisas relacionadas ao refinamento e testes da linguagem utilizada pela Enfermagem tornam-se necessárias, a fim de evidenciar o que tem sido aceito, rejeitado e modificado durante a utilização e comparação na prática dos termos existentes nas classificações de Enfermagem. É importante conhecer os termos já utilizados na prática clínica e aqueles ainda não constantes nas classificações a fim de que eles sejam incorporados às mesmas.

Além disso, cabe ainda ressaltar a necessidade de se descrever os elementos de áreas específicas da Enfermagem por possibilitar uma reflexão sobre a forma como estão sendo realizados os cuidados de enfermagem, por favorecer a melhoria da qualidade da assistência, e contribuir para a construção dos catálogos da $\operatorname{CIPE}^{\circledR}$, que podem ser incorporados em sistemas informatizados que auxiliem na operacionalização das etapas do processo de enfermagem na prática.

\section{REFERÊNCIAS}

1. Nóbrega MML, Garcia TR, Araruna JF, Nunes WCAN, Dias GKG, Beserra PJF. Mapeamento de termos atribuídos aos fenômenos de enfermagem nos registros dos componentes da equipe de enfermagem. Rev. Eletr. Enf. [Internet]. 2003 [cited $3009 \mathrm{dez}$ 30];(5)2:33-44. Available

from: http://www.fen.ufg.br/revista/revista5 2/pdf/mapa.pdf 2. Antunes MJM, Chianca TCM. As classificações de enfermagem na saúde coletiva: o projeto CIPESC. Rev Bras Enferm. 2002;55(6):644-51.

3. Bittencourt GKGD, Beserra PJF, Nóbrega MML, Garcia TR. Fenômenos identificados nos registros de enfermagem da clínica médica de um hospital de ensino. Revista Nursing 2005;88(8):432-35.

4. Charters KG. Nursing informatics, outcomes, and quality improvement. AACN Clin Issues. 2003;14(3):282-94.

5. Garcia TR, Nóbrega MML, Carvalho EC. Processo de enfermagem: aplicação à prática profissional. Online Braz J Nurs [Internet]. 2004 [cited 3009 dez 30];3(2): Available from: http://www.uff.br/nepae/siteantigo/objn302garciaetal.htm. 6. Nóbrega MML. Classificação Internacional para a prática de enfermagem é projeto do CIE. Revista Nursing. 2002;4(51):12-4.

7. Bittencourt GKGD, Nóbrega MML. Confirmação de significado para a prática profissional de termos atribuídos a ações de enfermagem: estudo descritivo. Online Braz ] Nurs [Internet]. 2006 [cited 3009 dez 30];5(3). Available from: http://www.uff.br/objnursing/index.php/nursing/article/ view/308/65.
8. Trigueiro EV, Lima MC, Araújo RTM, Nóbrega MML, Garcia TR. Definições teóricas de termos atribuídos a fenômenos de enfermagem identificados em prontuários clínicos de um hospital escola. Online Braz J Nurs [Internet]. 2007 [cited 3009 dez 30];6(0). Available from:

http://www.uff.br/objnursing/index.php/nursing/article/ view/630/148.

9. Pavel S, Nolet D. Manual de terminologia. Canadá: Departamento de Tradução do Governo Canadense; 2001.

10. Hickman JS. Introdução à teoria de enfermagem. In: George JB. Teorias de enfermagem: os fundamentos à prática profissional. $4^{\mathrm{a} e d .}$ Porto Alegre(RS): Artes Médicas Sul; 2000. p. 11-20

11. Nóbrega MML, Gutiérrez MGR. Método utilizado na adaptação transcultural da classificação de fenômenos de enfermagem da CIPE $^{\circledR}$ - Versão Alfa. Acta paul. enferm. $2001 ; 14(3): 44-51$.

12. Chianca TCMA. pesquisa em enfermagem e os sistemas de classificação dos elementos da prática. In: Tannure MC, Gonçalves AMP. Sistematização da Assistência de Enfermagem: Guia prático. Rio de Janeiro(RJ): Guanabara Koogan; 2007. p. 141-154

13. Marin HF. Vocabulário: recurso para construção de base de dados em enfermagem. Acta paul. enferm. 2000;13(1):86-9

14. Flaganan J, Jones DA. Nursing Language in a time of change: capturing the focus of the discipline. Int J Nurs Terminol Classif. 2007;18(1):1-2.

15. Conselho Internacional de Enfermeiros. Classificação Internacional para a Prática de Enfermagem Versão 1.0. Lisboa, Portugal: Ordem dos Enfermeiros de Portugal; 2005.

16. Garcia TR, Nóbrega MML. Processo de enfermagem e os sistemas de classificação dos elementos da prática profissional: instrumentos metodológicos e tecnológicos do cuidar. In: Anais do 70 Simpósio Nacional de Diagnósticos de Enfermagem; 2004; Belo Horizonte (MG), Brasil. Belo Horizonte (MG): ABEn; 2004. p.31-43. 17. Camiá GEK, Barbieri M, Marin HF. Fenômenos de enfermagem identificados em consultas de planejamento familiar segundo a ICNP - Versão Beta 2. Rev Latino-am Enfermagem [Internet]. 2006; [cited 2009 dez 30];14(5):674-81. Available from: http://www.scielo.br/pdf/rlae/v14n5/pt v14n5a06.pdf. 18. Tannure MC. Banco de termos da linguagem especial de Enfermagem para Unidade de Terapia Intensiva de adultos [dissertation]. Belo Horizonte (MG): Escola de Enfermagem/UFMG; 2008.

19. Lucena AF, Barros ALBL. Mapeamento cruzado: uma alternativa para a análise de dados em enfermagem. Acta paul. enferm. 2005;15(1):82-8.

Artigo recebido em 12.12.08.

Aprovado para publicação em 14.05.09.

Artigo publicado em 31.12.09. 